EPJ Web of Conferences 75, 06010 (2014)

DOI: 10.1051/epjconf/20147506010

(C) Owned by the authors, published by EDP Sciences, 2014

\title{
Influence of recording field direction on transition noise of stacked media
}

\author{
N. Tomiyama ${ }^{1,}$,, A. Oyama ${ }^{1}$, S. Sato ${ }^{1}$, and R. Sugita ${ }^{1}$ \\ ${ }^{1}$ Ibaraki Univ., 4-12-1 Nakanarusawa-cho, Hitachi, Ibaraki 316-8511, Japan
}

\begin{abstract}
Influence of recording filed direction on transition noise of stacked media was investigated by using magnetic printing for recording and MFM images. Stray field fluctuation along the cross-track direction in the transition area and dispersion of the transition position of the recorded medium decreased as the applied field angle increased from the perpendicular direction. This shows that the transition noise decreases with increasing in-plane component of the recording filed. On the other hand, in the transition area domain size which corresponds to half of the period of the stray field fluctuation was fixed near $50 \mathrm{~nm}$ regardless of the applied field direction.
\end{abstract}

\section{Introduction}

Reducing the transition noise is essential for increasing the areal density of hard disks (HDs) [1], [2]. Perpendicular HDs have been developed by mainly using stacked media and single pole heads with a trailing shield. The recording layer of stacked media consists of a capping layer of CoPt based alloy and a CoPt based granular layer [3]. Recording heads with a trailing shield enhance the magnetization reversal of the recording layer due to increase of in-plane component of magnetic field [4]. However, relationship between the in-plane component of recording field and the transition noise has not been elucidated yet. Previously, we clarified that domain structure in demagnetized media depends on the applied field direction for demagnetization, and that demagnetization with an in-plane field causes sub-domain structure, with consequent noise reduction [5], [6]. In this study, we experimentally investigate the influence of recording field direction, namely the in-plane component of recording field on the transition noise of the stacked media.

\section{Experimental}

Signals were recorded onto an HD by magnetic printing [7]. A schematic illustration of recording process is shown in Fig. 1. Recording process in this study is as follows. First, the recording layer of the HD is initially magnetized by an external magnetic field to the perpendicularly upward direction to the disk plane. Then, the HD is in contacted with a master medium, and a magnetic field $H_{\mathrm{a}}$ is applied. The magnetic flux concentrates into the contact area with the magnetic film of the master, while the magnetic field is weakened at the non-contact

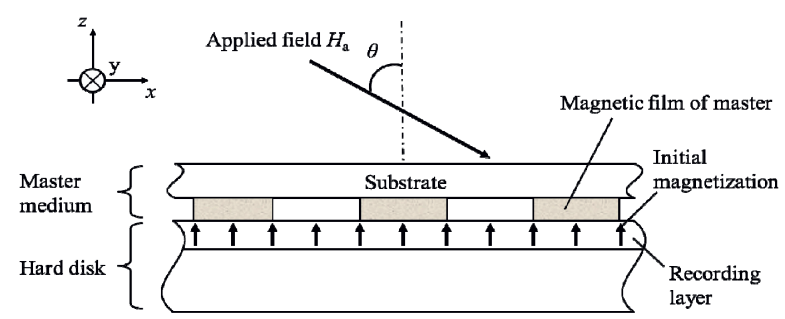

Fig. 1. Schematic illustration of recording process.

area. Finally, signal patterns are printed onto the recording layer. The angle $\theta$ of the $H_{\mathrm{a}}$ was varied from $0^{\circ}$ (perpendicular) to $90^{\circ}$ (in-plane). In order to bring the recording field distribution of the printing near a single pole head without the trailing shield (SPH) and a head with the trailing shield (TSH), magnetic film of master media was prepared as follows. For the $\theta$ of $0^{\circ}$ and $30^{\circ}$, the master medium with a $70 \mathrm{~nm}$ thick CoPt magnetic film was used, which is considered to nearly correspond to the SPH. For the $\theta$ of $60^{\circ}$ and $90^{\circ}$, the master medium with a $20 \mathrm{~nm}$ thick FeCo magnetic film was used, which is considered to nearly correspond to the TSH. Each master medium consisted of a line and space pattern. The bit length in each master medium was approximately 100 nm. A commercial HD, which has recording density of $700 \mathrm{~Gb} / \mathrm{in}^{2}$, was used, whose coercivity $H_{\mathrm{c}}$ was $5.2 \mathrm{kOe}$. The recorded HD was observed by using magnetic force microscopy (MFM, PNI products NanoR) and transition noise was investigated from MFM images.

\section{Results and discussion}

Figs. 2-5 show the MFM images for the HD recorded at various applied field angles $\theta$. The lower panel of each

\footnotetext{
${ }^{\mathrm{a}}$ Corresponding author: 13nm6691@hcs.ibaraki.ac.jp
} 

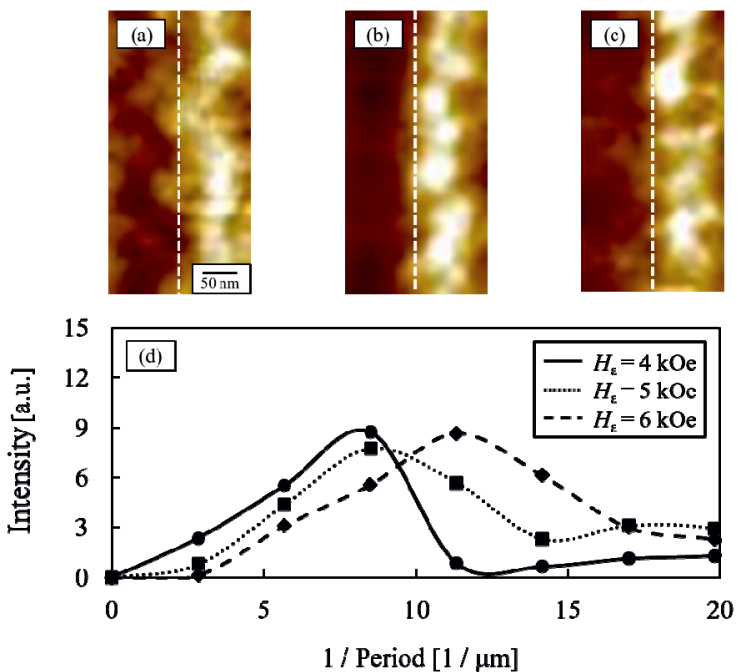

Fig. 2. MFM images near transition area and FFT spectra of MFM output at $\theta=0^{\circ}$. (a) MFM image of the HD recorded with $H_{\mathrm{a}}$ of $4 \mathrm{kOe}$, (b) $5 \mathrm{kOe},(\mathrm{c}) 6 \mathrm{kOe}$, and (d) FFT spectra along the broken lines in (a)-(c).
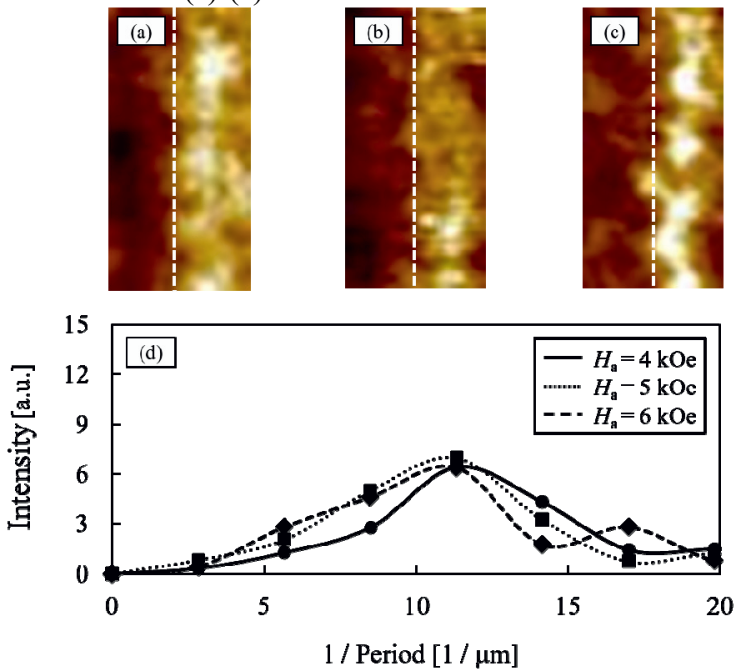

Fig. 3. MFM images near transition area and FFT spectra of MFM output at $\theta=30^{\circ}$. (a) MFM image of the HD recorded with $H_{\mathrm{a}}$ of $4 \mathrm{kOe}$, (b) $5 \mathrm{kOe}$, (c) $6 \mathrm{kOe}$, and (d) FFT spectra along the broken lines in (a)-(c).
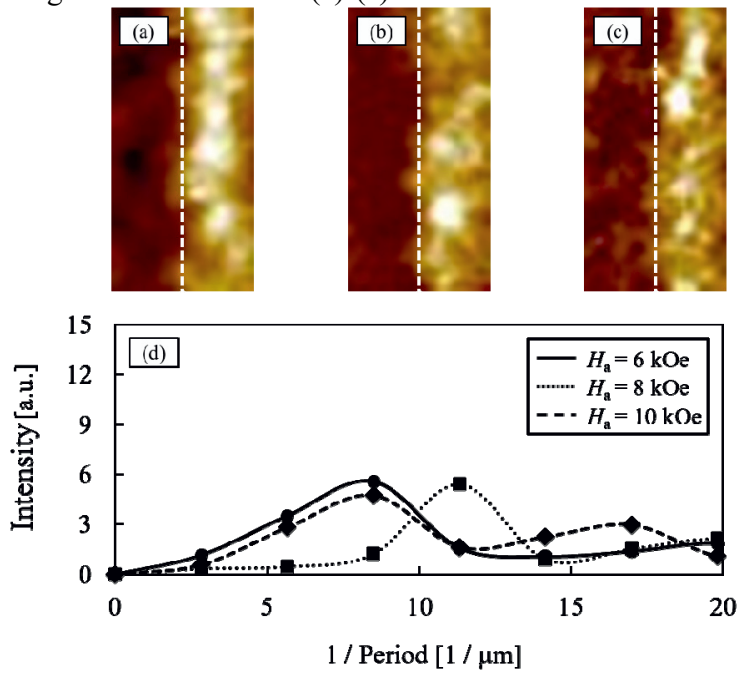

Fig. 4. MFM images near transition area and FFT spectra of MFM output at $\theta=60^{\circ}$. (a) MFM image of the HD recorded with $H_{\mathrm{a}}$ of $6 \mathrm{kOe}$, (b) $8 \mathrm{kOe}$, (c) $10 \mathrm{kOe}$, and (d) FFT spectra along the broken lines in (a)-(c).
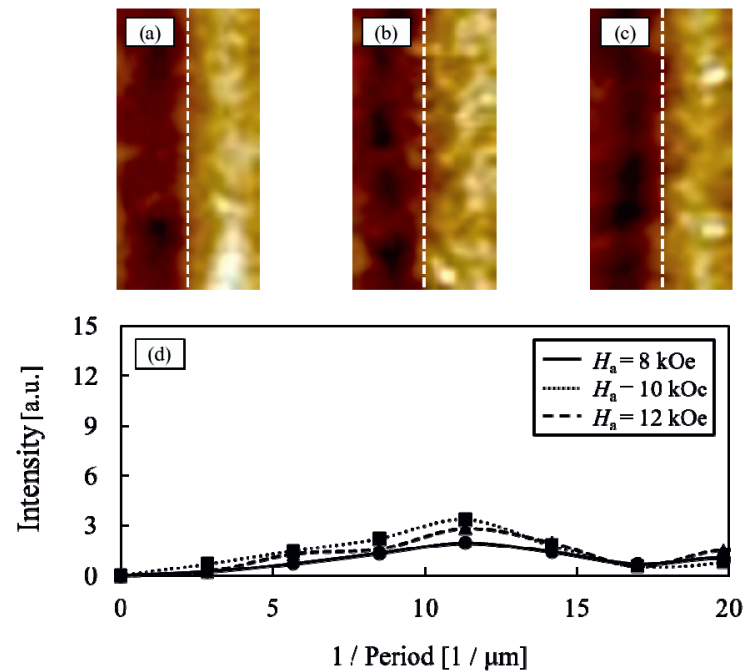

Fig. 5. MFM images near transition area and FFT spectra of MFM output at $\theta=90^{\circ}$. (a) MFM image of the HD recorded with $H_{\mathrm{a}}$ of $8 \mathrm{kOe}$, (b) $10 \mathrm{kOe}$, (c) $12 \mathrm{kOe}$, and (d) FFT spectra along the broken lines in (a)-(c).

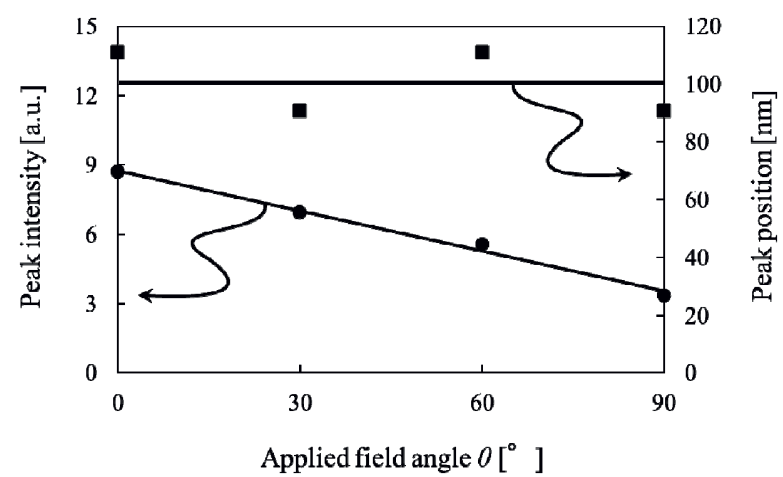

Fig. 6. Peak intensity and peak position of FFT spectra in Figs. $2-5$ as functions of angle of applied field.

figure is the Fast Fourier Transform (FFT) spectra along broken lines of the MFM images. The bright and dark areas in the MFM images represent stray fields of upward and downward direction, respectively. From the FFT spectra in Figs. 2-5, peak intensity decreases as $\theta$ increases from $0^{\circ}$ to $90^{\circ}$, being minimized at $\theta=90^{\circ}$. On the other hand, peak position remains constant as $\theta$ changes. In other words, domain size of cross-track direction remains unaffected as $\theta$ changes.

Fig. 6 is the dependence of the peak intensity and the peak position of the FFT spectra on applied field angles $\theta$ obtained from Figs. 2-5. In Fig. 6 the highest peak value for each $\theta$ was plotted. From Fig. 6 , it is found that the peak intensity at $\theta=0^{\circ}$ is approximately three times as that at $\theta=90^{\circ}$. On the other hand, in all FFT spectra, the period of the peaks is about $100 \mathrm{~nm}$. This means that the domain size along cross-track direction is approximately $50 \mathrm{~nm}$, and that domain size does not change as $\theta$ changes from $0^{\circ}$ to $90^{\circ}$ at MFM resolution. This result reflects the relationship between the applied magnetic field angle for demagnetization and demagnetized domain structure [5], [6]. This respect will be discussed in more detail later.

Fig. 7 shows the distribution of transition position measured along the cross-track direction. Namely, this figure indicates the frequency of transition positions 


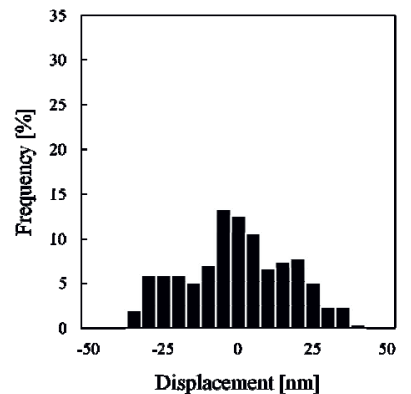

(a) $\theta=0^{\circ}$

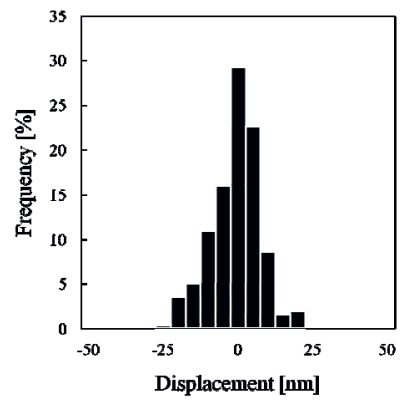

(c) $\theta=60^{\circ}$

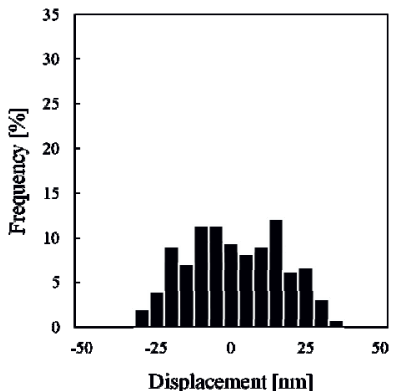

(b) $\theta=30^{\circ}$

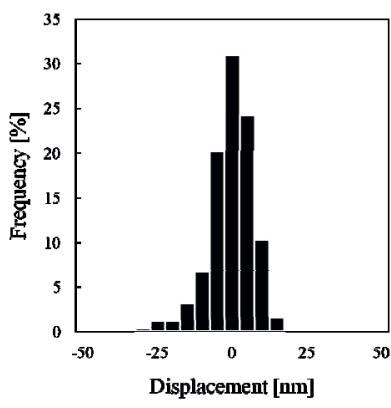

(d) $\theta=90^{\circ}$
Fig. 7. Distribution of transition position measured along the cross-track direction. (a) Applied field angle $\theta=0^{\circ}$, (b) $30^{\circ}$, (c) $60^{\circ}$, and (d) $90^{\circ}$.

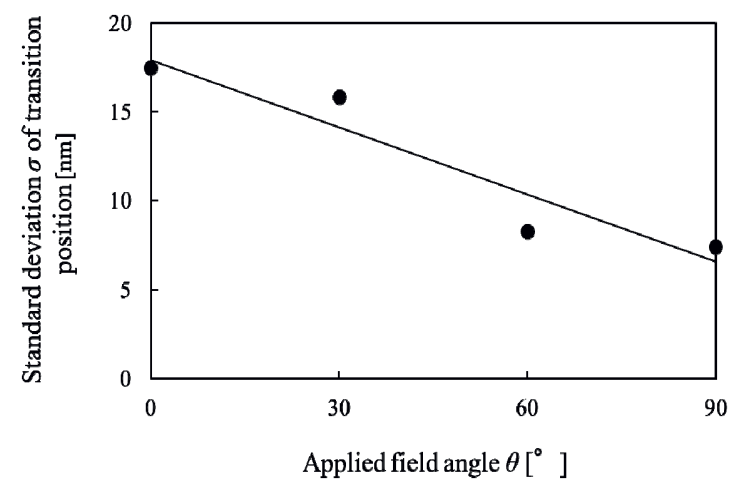

Fig. 8. Standard deviation $\sigma$ of transition position as a function of applied field angle $\theta$.

measured for each strip divided into $5 \mathrm{~nm}$ wide strip in the cross-track direction in the MFM images shown in Figs. 2-5. Here the average transition position is set to zero. Fig. 8 shows the relationship between standard deviation $\sigma$ of the transition position obtained from Fig. 7 and the applied field angle $\theta$. In Fig. 8, $\sigma$ decreases as $\theta$ increases, being minimized at $\theta=90^{\circ}$. Therefore, the transition width is the narrowest at $\theta=90^{\circ}$. From Figs. 28 , we conclude that the transition noise decreases as the in-plane component of the recording field increases.

As mentioned above, here we investigated the transition noise for magnetic printing. To associate magnetic printing as head recording, we calculated the magnetic field distribution at various applied field angles near the transition area.

Fig. 9 shows the distribution of the perpendicular component $H_{\mathrm{z}}$ and the in-plane component $H_{\mathrm{x}}$ of the magnetic field at the position in recording layer near the

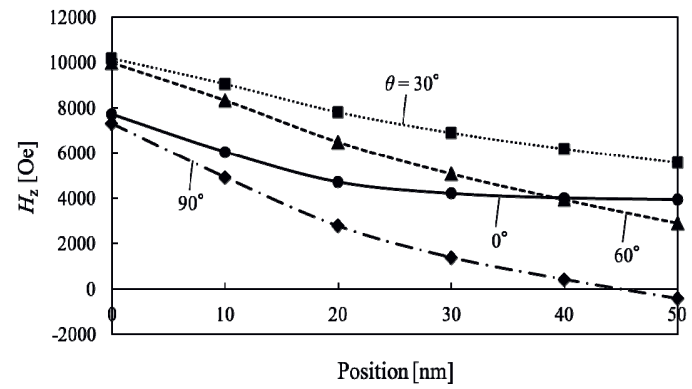

(a) $H_{2}$

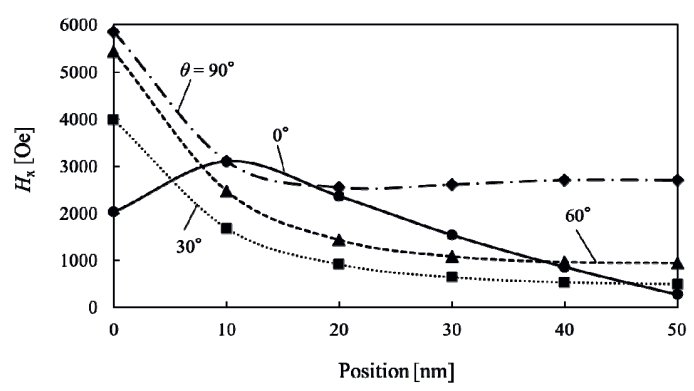

(b) $H_{x}$

Fig. 9. Calculated magnetic field at the position in recording layer for magnetic printing.

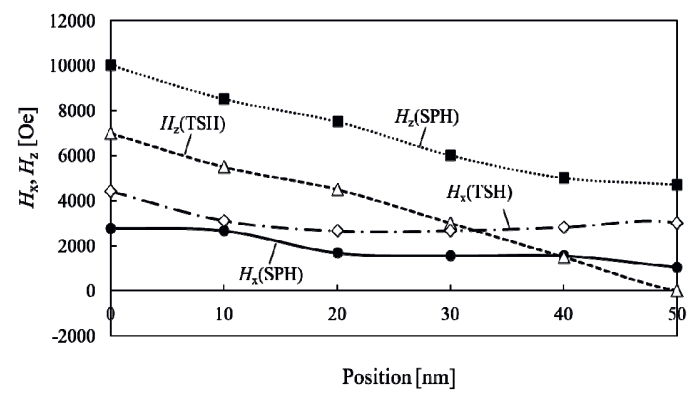

Fig. 10. Recording field at the position in recording layer for SPH and TSH.

transition area for $\theta$ of $0^{\circ}, 30^{\circ}, 60^{\circ}$, and $90^{\circ}$. The field distribution of the SPH and the TSH is plotted in Fig. 10 [8]. Near the transition area, the field distribution at $\theta=$ $30^{\circ}$ is almost the same as that of the SPH, while at $\theta=$ $90^{\circ}$, it is almost the same as that of the TSH. Therefore, we conclude that the transition noise for the SPH and the TSH can be inferred from Figs. 2-8, and the transition noise is reduced in the TSH relative to the SPH.

The reason why the transition noise is reduced by increasing the in-plane component of the recording field is presumed as follows. Fig. 11 shows MFM images and MFM output for the medium demagnetized with fields applied at different angles $\theta_{\mathrm{d}}$ [5]. Domain size is essentially unchanged as $\theta_{\mathrm{d}}$ varies from $0^{\circ}$ to $90^{\circ}$. On the other hand, the MFM output decreases as $\theta_{\mathrm{d}}$ increases, presumably because of domain including sub-domains less than the MFM resolution [5], [6]. It is inferred that the transition noise experimentally evaluated in this study reflects the domain structure of the demagnetized stacked media, and that decrease of transition noise originates from increasing sub-domains in the transition areas as the in-plane component of recording field increases. 

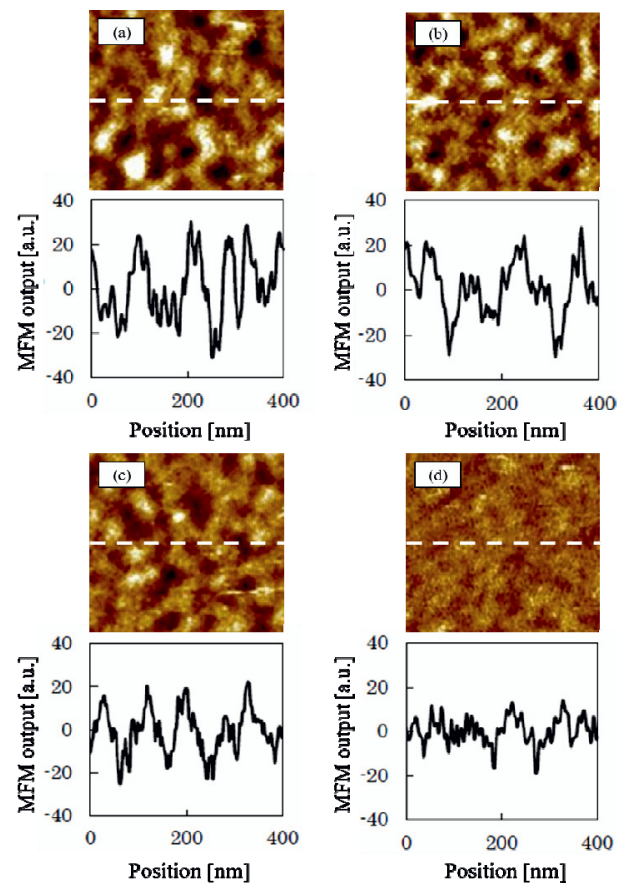

Fig. 11. MFM images of a stacked medium demagnetized at field angle $\theta_{\mathrm{d}}$ of (a) $0^{\circ}$ (perpendicular), (b) $30^{\circ}$, (c) $60^{\circ}$, and (d) $90^{\circ}$ (in-plane). Each bottom panel is the MFM output [5].

\section{Conclusion}

In this study, we investigated the influence of record- ing field direction on the transition noise of stacked media by using the magnetic printing for recording. Both the stray field fluctuation along the cross-track direction in the transition area and the dispersion of the transition position of the recorded medium decreased as the applied field angle increased from the perpendicular direction. This shows that the transition noise decreases with increasing in-plane component of the recording field. On the other hand, in the transition area domain size which corresponds to half of the period of the stray field fluctuation was fixed near $50 \mathrm{~nm}$ regardless of the applied field direction. The applied field direction dependence of the strength and the period of fluctuation in the stray field from transition area coincides with demagnetizing field direction dependence of the stray field from the demagnetized media. From this result, it is inferred that the decrease of transition noise originates from increasing sub-domains in the transition areas as the in-plane component of the recording filed increases.

\section{Acknowledgment}

This work was supported in part by the Grant-in-Aid for Scientific Research (C) (No. 24560394) from the Japan Society for the Promotion of Science (JSPS) of Japan.

\section{References}

1. K. Miura, H. Muraoka, Y. Sugita, and Y. Nakamura: J. Magn. Soc. Jpn., 24, 231 (2000).

2. H. Sohn and R.H. Victora: IEEE Trans. Magn., 49, 824 (2013).
3. J. Ariake, S. Watanabe, and N. Honda: IEEE Trans. Magn., 43, 2304 (2007).

4. D. Z. Bai, P. Luo, A. Torabi, D. Terrill, J. Wang, K. Stoev, F. Liu, M. Moneck, Y. Tang, and J. G. Zhu: IEEE Trans. Magn., 43, 600 (2007).

5. S. Sato, Y. Yamaguchi, T. Komine, and R. Sugita: IEEE Trans. Magn., 48, 3181 (2012).

6. Y. Yamaguchi, S. Sato, T. Komine, and R. Sugita: IEEE Trans. Magn., 49, 3584 (2013).

7. N. Sheeda, M. Nakazawa, H. Konishi, T. Komine, and R. sugita: IEEE Trans. Magn., 45, 3676 (2009).

8. K. Takano, M. Sakai, J. Smyth, and M. Dovek: IEEE Trans. Magn., 43, 594 (2007). 